\title{
The Effect of Spinal Immobilization on Vital Signs
}

\author{
Christopher R. Foerster, MSc
}

Lambton EMS, Lambton County, Canada and University of Queensland, Brisbane, Australia

Correspondence:

Christopher R. Foerster, MSc

Lambton EMS

Lambton County, Ontario, Canada

E-mail: christopher.foerster@gmail.com

Received: April 11, 2013

Accepted: April 22, 2013

Online publication: June 19, 2013

doi:10.1017/S1049023X13008674
Bruijns et al describe an intriguing study in "Effect of Spinal Immobilization on Heart Rate, Blood Pressure and Respiratory Rate" published in Prehospital and Disaster Medicine. ${ }^{1}$ It is important that we continue to examine the effects of spinal immobilization on our patients, and the authors' study is an important contribution to this. Unfortunately, the authors have drawn a far broader conclusion than is supported by their work.

The authors' conclusion is that "abnormal vital signs in a clinical situation should not be considered to be the result of immobilization." This conclusion is unsupported by the study for two reasons. First, the subjects are unrepresentative of patients attended to by paramedics. These patients tend to be older and with preexisting medical problems, while the subjects in this study are young (with a mean age of 37) and free of cardiac or respiratory problems.

Second, the conclusion of the study ignores the effect that anxiety might have on the vital signs of patients undergoing spinal immobilization. All subjects in this study were emergency department staff or paramedic students who were familiar with the spinal immobilization procedure. The authors describe this as being "purposeful in order to reduce the possible confounding effect of anxiety" on vital signs. This fits with the authors' stated goal of "establish[ing] whether the pain and discomfort associated with spinal immobilization and the maneuvers commonly used in injured patients (eg, log roll) affect the HR, BP and RR" (my emphasis). But whether or not the pain and discomfort experienced during spinal immobilization causes vital sign changes, the anxiety associated with the procedure might. The well-documented "white coat syndrome" manifestation of anxiety by vital sign changes ${ }^{2}$ could have a parallel in the prehospital setting. It is possible that the effect of a cervical collar and backboard could be akin to that of a doctor appearing in a consultation room.

What the authors have shown is that in young and healthy subjects who are familiar with spinal immobilization, the pain and discomfort caused by the procedure do not lead to vital sign changes. Given the potential effect of anxiety on vital signs, as well as the fact that patients seen in the prehospital setting are often older and with comorbidities, it would be helpful to see a similar study with more realistic subjects in a more realistic setting. This will enable us to draw conclusions that are relevant to clinical practice.

References

1. Bruijns SR, Guly HR, Wallis LA. Effect of spinal immobilization on heart rate, blood pressure and respiratory rate. Prehosp Disaster Med. 2013;28(2):1-5.

2. Mancia G, Bertinieri G, Grassi G, et al. Effects of blood-pressure measurement by the doctor on patient's blood pressure and heart rate. Lancet. 1983;2(8352):695-698. 


\section{Author reply:}

\section{Stevan R. Bruijns, FCEM; ${ }^{1,2}$ Henry R. Guly, FCEM; ${ }^{2}$ Lee A. Wallis, MD ${ }^{1}$}

1. Division of Emergency Medicine, University of Capetown, Cape Town, South Africa

2. Department of Emergency Medicine, Derriford Hospital, Plymouth, United Kingdom

\section{Correspondence:}

Stevan R. Bruijns, FCEM

Emergency Department

Derriford Hospital

Plymouth PL6 8RY United Kingdom

E-mail: stevan.bruijns@afjem.com

\author{
Abbreviations: \\ HR: heart rate \\ SBP: systolic blood pressure
}

Mr. Foerster summarizes the limitations of our paper accurately; small samples limit the interpretation of results which is why these are usually followed by larger studies. We would like to draw Mr. Foerster's attention to another recent publication that describes similar findings with regards to spinal immobilization in a study of injured patients comparing heart rate (HR) and systolic blood pressure (SBP) between an immobilized and non-immobilized cohort. ${ }^{1}$ There was no significant relationship between spinal immobilization and HR or SBP. Interestingly, in another part of this study, it was found that the HR of injured patients was 10 beats per minute higher than that of an uninjured cohort and that this was irrespective of age. The SBP did not replicate this pattern, with almost no significant difference found between the two groups. It is not quite clear why the white coat effect (defined as a SBP increase of between $25-30 \mathrm{mmHg}$ and HR increase of more than 10 beats per minute) described elsewhere is not observed in association with injury. Previous studies that have looked at the effect of emergency transport on vital signs found a significant effect only in experimental studies, ${ }^{2,3}$ whilst none was observed when studying an actual patient group. ${ }^{4}$ It is already known that the physiological response due to hemorrhage alone disappears when tissue injury co-exists. ${ }^{5}$ It is likely that injury has a direct effect on vital signs which also attenuates the fight-andflight response triggered by anxiety related to immobilization and transport. Clearly further research is needed to establish this interesting pathophysiology. The clinical bottom line is that where injury is concerned, anxiety does not seem to affect vital signs significantly. Therefore in the presence of injury, instead of assuming that abnormal vital signs are due to a physiological process, it would be more prudent to first consider a pathological one.

\footnotetext{
References

1. Bruijns SR, Guly HR, Bouamra O, Lecky F, Wallis LA. Heart rate and systolic blood pressure in patients with minor to moderate, non-haemorrhagic injury versus normal controls. Emerg Med J. doi:10.1136/emermed-2012-201760.

2. Dörges V WV, Kühl A, Dix S, et al. Emergency medical service transport-induced stress? An experimental approach with healthy volunteers. Resuscitation. 2001;49(2):151-157.

3. Witzel K. Effects of emergency ambulance transportation on heart rate, blood pressure, corticotropin, and cortisol. Ann Emerg Med. 1999;33(5):598-599.

4. Weber U, Reitinger A, Szusz R, Hellmich C, Steinlechner B, Hager H, et al. Emergency ambulance transport induces stress in patients with acute coronary syndrome. Emerg Med J. 2009;26(7):524-528.

5. Foex B. Systemic responses to trauma. Br Med Bull. 1999;55(4):726-743.
} 